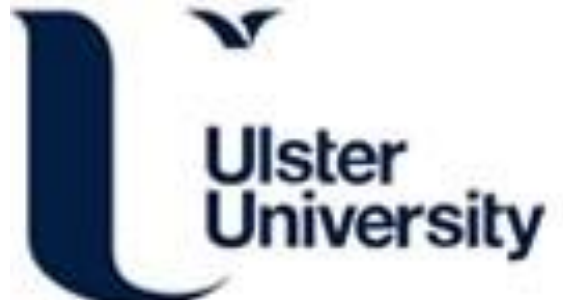

\section{An Automatic Subject Specific Intrinsic Mode Function Selection for Enhancing Two- Class EEG based Motor Imagery-Brain Computer Interface}

Gaur, P., Pachori, R. B., Wang, H., \& Prasad, G. (2019). An Automatic Subject Specific Intrinsic Mode Function Selection for Enhancing Two-Class EEG based Motor Imagery-Brain Computer Interface. IEEE Sensors Journal, 19(16), 6938-6947. [8695803]. https://doi.org/10.1109/JSEN.2019.2912790

Link to publication record in Ulster University Research Portal

Published in:

IEEE Sensors Journal

Publication Status:

Published (in print/issue): 15/08/2019

DOI:

10.1109/JSEN.2019.2912790

Document Version

Author Accepted version

\section{General rights}

Copyright for the publications made accessible via Ulster University's Research Portal is retained by the author(s) and / or other copyright owners and it is a condition of accessing these publications that users recognise and abide by the legal requirements associated with these rights.

\section{Take down policy}

The Research Portal is Ulster University's institutional repository that provides access to Ulster's research outputs. Every effort has been made to ensure that content in the Research Portal does not infringe any person's rights, or applicable UK laws. If you discover content in the Research Portal that you believe breaches copyright or violates any law, please contact pure-support@ulster.ac.uk. 


\title{
An Automatic Subject Specific Intrinsic Mode Function Selection for Enhancing Two-Class EEG based Motor Imagery-Brain Computer Interface
}

\author{
Pramod Gaur, Ram Bilas Pachori, Member, IEEE, Hui Wang, Senior Member, IEEE, \\ and Girijesh Prasad, Senior Member, IEEE
}

\begin{abstract}
The electroencephalogram (EEG) signals tend to have poor time-frequency localization when analysis techniques involve a fixed set of basis functions such as in short-time Fourier transform (STFT) and wavelet transform (WT). These signals also exhibit highly non-stationary characteristics and suffer from low signal-to-noise ratio (SNR). As a result, there is often poor task detection accuracy and high error rates in designed braincomputer interfacing (BCI) systems. In this paper, a novel preprocessing method is proposed to automatically reconstruct the EEG signal by selecting the intrinsic mode functions (IMFs) based on a median frequency measure. Multivariate empirical mode decomposition (MEMD) is used to decompose the EEG signals into a set of IMFs. The reconstructed EEG signal has high SNR and contains only information correlated to a specific motor imagery task. The common spatial pattern (CSP) is used to extract features from the reconstructed EEG signals. The linear discriminant analysis (LDA) and support vector machine (SVM) have been utilized in order to classify the features into left hand motor imagery (LHMI) and right hand motor imagery (RHMI) tasks. Our experimental results on the BCI competition IV dataset $2 \mathrm{~A}$ show that the proposed method with fifteen channels outperforms bandpass filtering with twenty-two channels $(>1 \%)$ and by $>9 \%(p=0.0078)$ with raw EEG signals, $>13 \%(p=0.0039)$ with empirical mode decomposition (EMD) based filtering and $>17 \%(p=0.0039)$ with discrete wavelet transform (DWT) based filtering.
\end{abstract}

Index Terms-BCI, MEMD, filtering, common spatial pattern, linear discriminant analysis.

\section{INTRODUCTION}

A brain-computer interface (BCI) is a system which facilitates a means of communication with external assistive devices utilizing brain signals such as electroencephalogram (EEG) [1]. In BCI, the aim is to translate the intent of a user into control command by EEG signals for a neuroprosthetics or a computer application. A popular example for a $\mathrm{BCI}$ modality is motor imagery (MI) based BCI [2], [3]. The user is expected to imagine the execution of a movement for a particular limb. Moreover, a rhythmic activity is seen in the sensorimotor cortex of the brain for a specific movement in MI-based BCI [4], [5]. The BCI systems identify these rhythmic activities and translate them into desired command. One of the major problems in EEG based BCI systems is the non-stationarity which arises when EEG signals are originating from different sources. In addition, the recorded EEG signals have a low signal-to-noise ratio (SNR)[6]. The low SNR may be due to artifacts resulting from electrooculogram (EOG) or electromyogram (EMG) interference and electrical power lines.
To increase the SNR, a useful step would be to remove these distortions or artifacts from raw EEG signals before extracting the features for classification [1]. An extension method based on common spatial pattern (CSP) has been studied to handle the adverse results of intervention from noisy EEG signals [7]. A Bayesian learning method has been implemented for spatial filtering in [8] for handling EEG signals with extremely low SNR. The methods built on the self-organizing fuzzy neural network (SOFNN) and the neural network $(\mathrm{NN})$ concepts have also been proposed to attain better feature separation for MI tasks in MI-BCI [9], [10], [11]. Recently, a filtering technique based on quantum neural network has been proposed before the feature extraction step in [5] to gain better separation between classes. However, a univariate empirical mode decomposition (EMD) technique is also well suited for the analysis of non-stationary and non-linear signals [12], [13], [14]. This method is data dependent and adaptive in nature. It gives a group of intrinsic mode functions (IMFs). These are considered as narrow-band amplitude and frequency modulated (AFM) signals. Univariate EMD, however suffers from the problem of mode-mixing wherein similar frequencies occur in different IMFs [15]. To overcome this issue, a multichannel version namely, multivariate EMD (MEMD) has been investigated to show its comparative advantage [16], [15], [17], [18]. The MEMD allows to achieve high localization of information pertaining to specific frequency-bands. It decomposes the raw EEG signal into a finite set of frequency modulated (FM) and amplitude modulated (AM) components known as multivariate IMFs (MIMFs) [15]. It also provides the same number of IMFs for all the data channels in the time domain. It should be noted that the original MEMD decomposition method used visual inspection to discard the MIMFs [15]. Recently, a research group investigated several popular signal processing techniques, namely, EMD, discrete wavelet transform (DWT) [19] and wavelet packet decomposition (WPD) to classify multi-channel EEG signals into two classes ([20]. Moreover, EMD and DWT have been studied on a single ECG channel to extract respiratory waveforms [21].

In this work, a novel way to automatically select the subject specific MIMFs is proposed. The selected MIMFs are chosen based on the median frequency measure corresponding to mu and beta rhythms. These selected IMFs are summed to reconstruct EEG signal and remaining MIMFs are discarded. The detailed procedure for MIMF selection is provided by the algorithm II] discussed in Section [I]. 


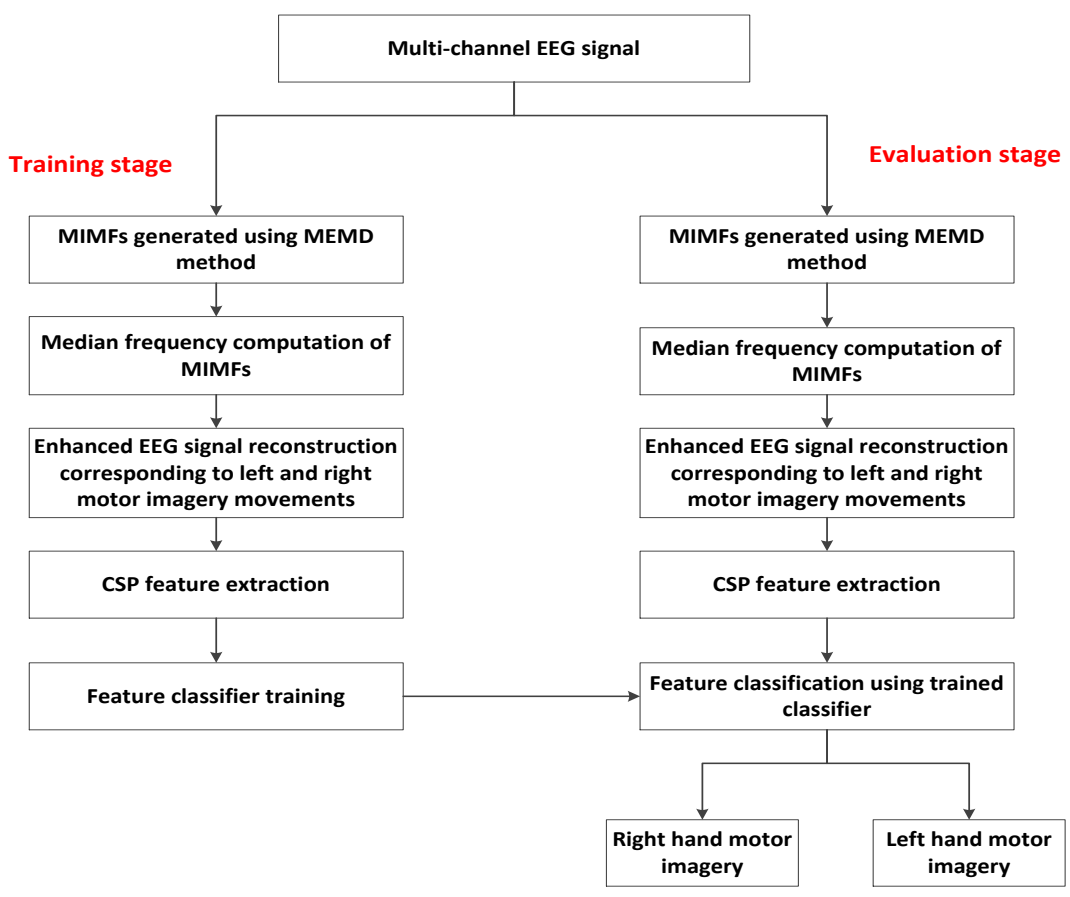

Fig. 1. Block diagram of the proposed methodology

Previously, a filtering technique was proposed [12] based on EMD which is restricted to decomposition of EEG signals on one channel at a time in MI-based BCI. Also, a multivariate extension of EMD based filtering was also proposed namely, MEMD based filtering [22], wherein mean frequency was utilized to identify the MIMFs with all the provided twentytwo channels but in this work only fifteen channels are used for studying the median frequency measure to automatically identify the subject specific MIMFs to reconstruct the enhanced EEG signals without compromising with the classification accuracy.

The aims of the paper are thus as follows:

1) To investigate the inter- and intra-subject nonstationarities persistent in the EEG signals;

2) To study whether the subjects have the different or same frequency components involved in MI task when EEG signals are measured from the same cortical areas;

3) Use median frequency measure to automatically find the subject specific MIMFs;

4) To find whether it is possible to achieve better classification accuracy using fewer monopolar EEG channels;

5) To report the classification accuracy when single trials are classified.

A block diagram representation of the proposed pipeline including MEMD based filtering with CSP features is shown in Figure 1. The remaining paper is organised as follows: In Section II a brief introduction about the MEMD technique is discussed. The CSP features and details about the LDA classifier are discussed in Section III] and Section IV. The results pertaining to the proposed method along with the

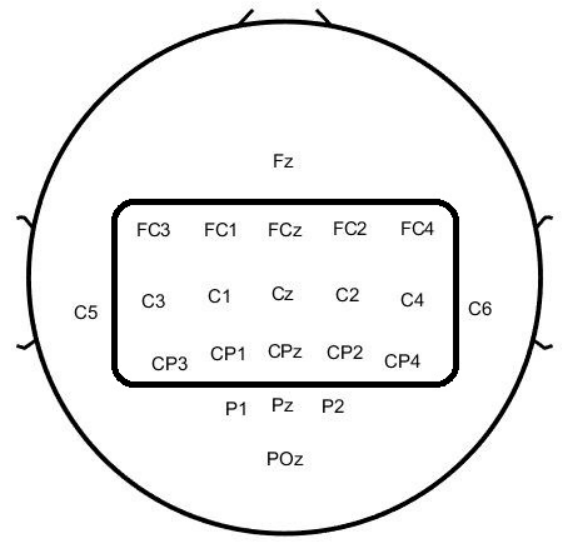

Fig. 2. Channels over the motor cortex used for the current study.

discussion on those results and comparison with the methodologies developed by other research groups are discussed in Section $\mathrm{V}$ and finally, the paper concludes in section VI

\section{Multivariate EMPIRICAL MODE DECOMPOSITION (MEMD)}

The EMD is a data driven technique to decompose a signal into a finite set of band limited basis functions called IMFs [23]. The MEMD has been developed recently where the local mean is computed by averaging the projection of the signal 
across multiple $n$-dimensional envelopes. The EEG signals tend to have poor SNR. They also suffer from interference from both EOG and EMG [24]. The EEG signals of interest corresponding to a particular movement (such as $\mu$ and $\beta$ rhythms for motor imagery) may contain a lot of noise which leads to erroneous results. Therefore, a preprocessing technique is required to filter out the noise without weakening the original signal. In 1998, Huang et al. proposed EMD [23] which decomposes the original signal into a finite group of band limited basis functions which are known as IMFs, represented as follows:

$$
Z(t)=\sum_{k=1}^{p} I_{k}(t)+R d_{p}(t)
$$

where $Z(t)$ denotes the actual signal in the time domain, $I_{k}(t)$ represents the $k^{\text {th }} \mathrm{IMF}$, and $R d_{p}(t)$ gives the residue. Thus, a summation of the selected IMFs can be done to reconstruct the signal of interest. Rest of the IMFs are discarded which may contribute to other artifacts and noise. However, univariate EMD suffers from the mode-mixing issue. To overcome this issue, another research group has proposed an ensemble empirical mode decomposition (EEMD) method [25]. Unfortunately, it is also not suitable for real-time implementations because it adds white noise to the signals and requires ensemble of many EMD methods which make this method very time-consuming. Further, a multichannel version of the EMD method has been proposed which utilizes cross channel information present across channels called MEMD [15], [26], [27], [28]. The mean $A(t)$ is computed through multivariate envelope curves, given as [29]:

$$
A(t)=\frac{1}{p} \sum_{j=1}^{p} \mathrm{e}^{\theta_{j}}(t)
$$

where $j$ gives the length of vectors. $\mathrm{e}^{\theta_{j}}(t)$ denotes the multivariate envelope curves for the entire set of direction vectors. Further, the candidate IMF $R d(t)$ by $R d(t)=Z(t)-A(t)$ is computed. If the candidate IMF satisfies the stoppage criterion, then it becomes the multichannel IMF. Otherwise, we set the input $Z(t)$ equal to the remainder $R d(t)$ and the complete process will be repeated again till remaining multivariate IMFs have been extracted. For more details refer to [15].

The contribution of this work is to automatically select the subject specific MIMFs for a particular subject corresponding to $\mu$ and $\beta$ rhythms and then perform the summation of selected MIMFs. In order to select the MIMFs, the median frequency measure has been calculated for all MIMFs corresponding to the LHMI and RHMI. The median frequency of each IMF is calculated as half of the total power of IMF in the frequency domain [30]. The mathematical expression of median frequency is given as,

$$
M D N F_{I M F}=\sum_{i=1}^{M D N F} P_{i}=\sum_{i=M D N F}^{n} P_{i}=\frac{1}{2} \sum_{i=1}^{n} P_{i}
$$

where $n$ denotes the length of frequency bin, and $P_{i}$ gives the total power of IMF in the frequency domain. These computed median frequencies of each IMF represent frequency at which the IMF power spectrum is divided into two regions having equal amplitude in the spectrum in frequency domain. The median frequency was used to first automatically identify the subject specific MIMFs providing major contribution to $\mu$ and $\beta$ rhythms. Thereafter, the selected MIMFs are summed to reconstruct the enhanced EEG signals corresponding to the LHMI and RHMI. The reconstructed EEG signals contains information which provides major contribution to $\mathrm{mu}(\mu)(8-12$ $\mathrm{Hz})$ and beta $(\beta)(16-24 \mathrm{~Hz})$ rhythms observed over the central region of the brain when the subjects plan or execute hand movements. The features extracted from the reconstructed EEG signals are used for classification of LHMI and RHMI tasks.

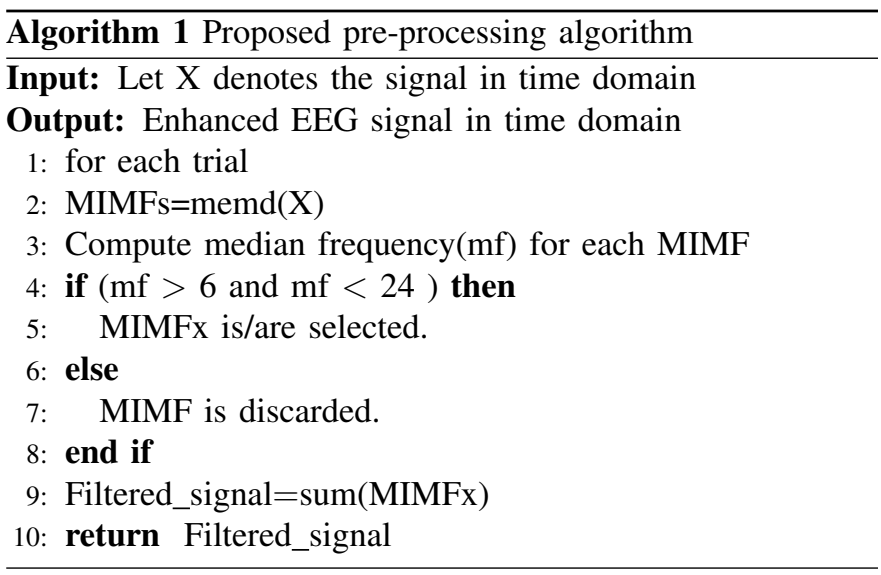

\section{COMMON SPATIAL PATTERN (CSP)}

CSP features are calculated by utilizing the CSP algorithm from selected fifteen channels corresponding to MI-based BCI ( Fig. 2). The CSP algorithm may be understood as a method which generates weight maps of the selected channels for EEG signals. These weight maps provide the importance of EEG signal content of the channels for separating the two conditions present in the data [31], [32]. These weight maps are spatial filters which are then projected onto data. With the projection of these spatial filters, the data is altered in such a way that the ratio of the variance for EEG amplitudes between the given two conditions is maximized. Therefore, the variance of the filtered EEG signal may serve as a discriminative feature for a two-class classification problem. The scalp potentials of the recorded EEG signal may not have good spatial resolution. One possible reason may be the volume conduction problem. With the poor spatial resolution, the EEG signal classification task becomes tougher if other sources give stronger signals when compared to the required signal in the specified frequency range [31].

As mentioned in Section I] the CSP algorithm has shown promising results in computing spatial filters for detecting event related desyncronization / event related synchronization (ERD/ERS) [31], [33]. It is a trial specific supervised decomposition of signals which is parameterized by a projection matrix $P M \in \Re^{C h n \times C h n}$ where $C h n$ denotes the number of channels selected. In EEG signal sensor space, $P M$ gives the 

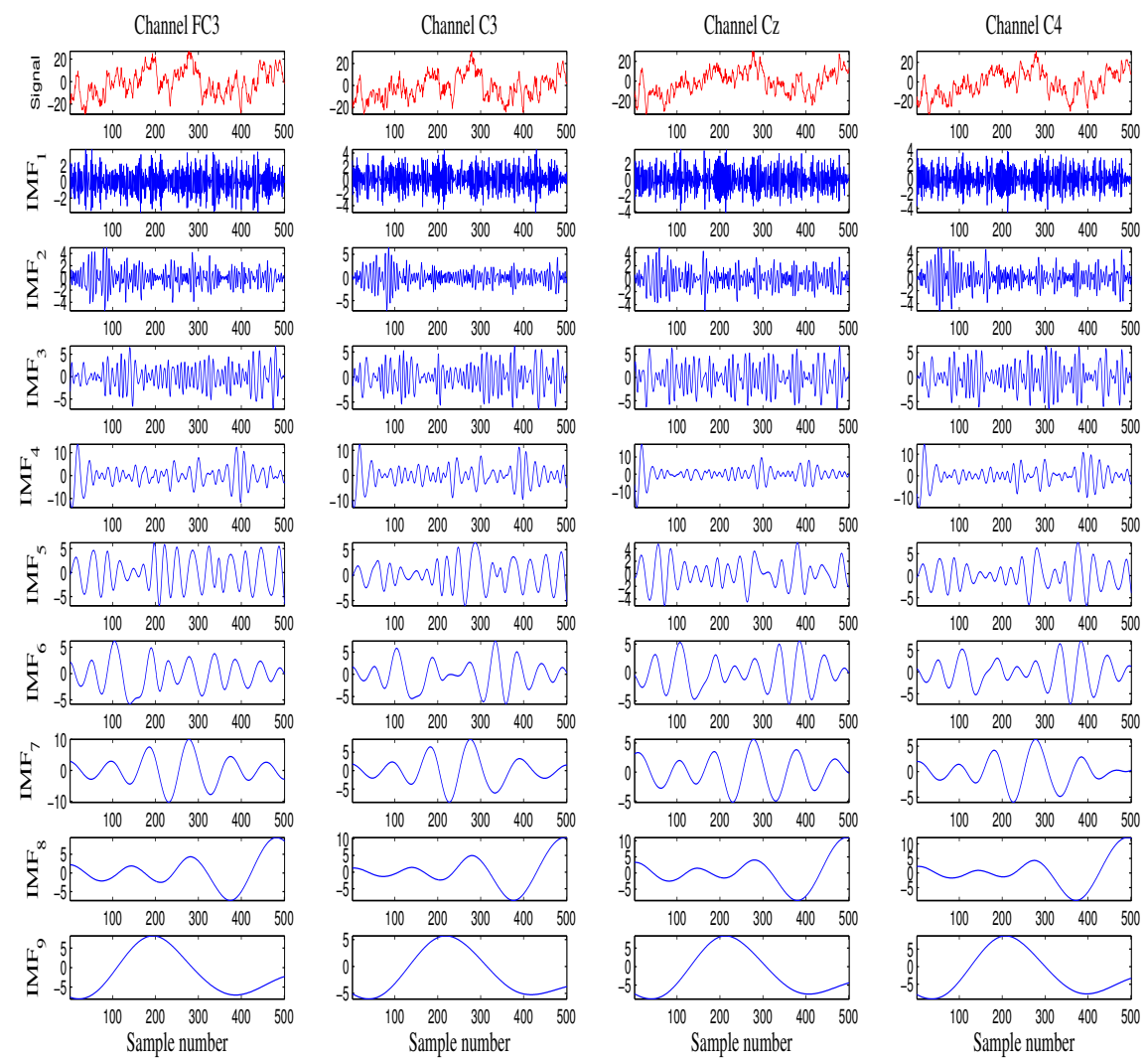

Fig. 3. The EEG signal corresponding to channels FC3, C3, Cz, and C4 of the trial 1 of A08T for the LHMI task and its first nine IMFs.

projection of a single trial $\operatorname{Tr} \in \Re^{C h n \times t}$ to $C \in \Re^{C h n \times t}$ in the surrogate sensor space, which is represented as:

$$
C=(P M)^{T} \times T r
$$

where $\operatorname{Tr}$ gives $C h n \times t$ EEG measurement data from a single trial, obtained from $C h n$ EEG channels sampled $t$ times. The spatial filters are denoted by the rows of $P M$.

The spatially filtered signal $C$ provided in (4), maximizes the ratio of the variances of the two classes. A CSP analysis is employed to obtain an efficient discrimination between two different conditions which are described by ERD/S phenomena. However, the variances concerning to a small subset of spatial filters are usually selected. The first $M$ and last $M$ rows of $C$ i.e., $C_{e}, e \in\{1,2, \ldots, 2 M\}$ given in (4) are used. In this study, we have considered $M=5$ spatial filters. For more details, refer to [31].

\section{ClassificATion}

Generally, it is a demanding task to find the best feature combination which can reduce classification errors and provide better feature separability [34]. The LDA classifier and SVM classifier with linear kernel have been applied in this work, which is most popularly used on EEG signals for BCI applications. It reduces the dimensionality of the feature set and also preserves the maximum information required for class discrimination.

\section{RESULTS AND DISCUSSION}

The BCI competition IV dataset 2A [33], [35] has been used for this study. This dataset contains EEG signals recorded from nine healthy subjects, namely, A01-A09 for left hand, right hand, feet, and tongue MI tasks. The effectiveness of the proposed preprocessing technique has been evaluated on LHMI and RHMI tasks in all nine subjects. Each subject's EEG data is recorded over two sessions, e.g., A01T and A01E [33], [35]. In this paper, only fifteen channels (i.e., FC3, C3, $\mathrm{CP} 3, \mathrm{FC} 2, \mathrm{C} 2, \mathrm{CP} 2, \mathrm{FC} 1, \mathrm{C} 1, \mathrm{CP} 1, \mathrm{FCz}, \mathrm{Cz}, \mathrm{CPz}, \mathrm{FC} 4$, $\mathrm{C} 4$ and $\mathrm{CP} 4)$ are considered for analysis from the available twenty-two channels as shown in Figure 2 More details on this dataset can be obtained from [35]. For the computation of classification accuracy (in \%) for each subject, 100\% of A0ST data has been considered for training the classifier model using an LDA classifier. Then, it is evaluated on $100 \%$ data A0SE of the evaluation session, where $S$ represents the subject number. In the MI paradigm, the MI task begins at 2 second; the training session and evaluation session features have been extracted from the 2.5 to 4.5 seconds time interval similar to the competition winner [33].

During the training session, a five-fold cross-validation has been applied to classify the EEG signals into LHMI and RHMI tasks. To demonstrate the decomposition dynamics of the MEMD technique, single trial EEG signals per class are 

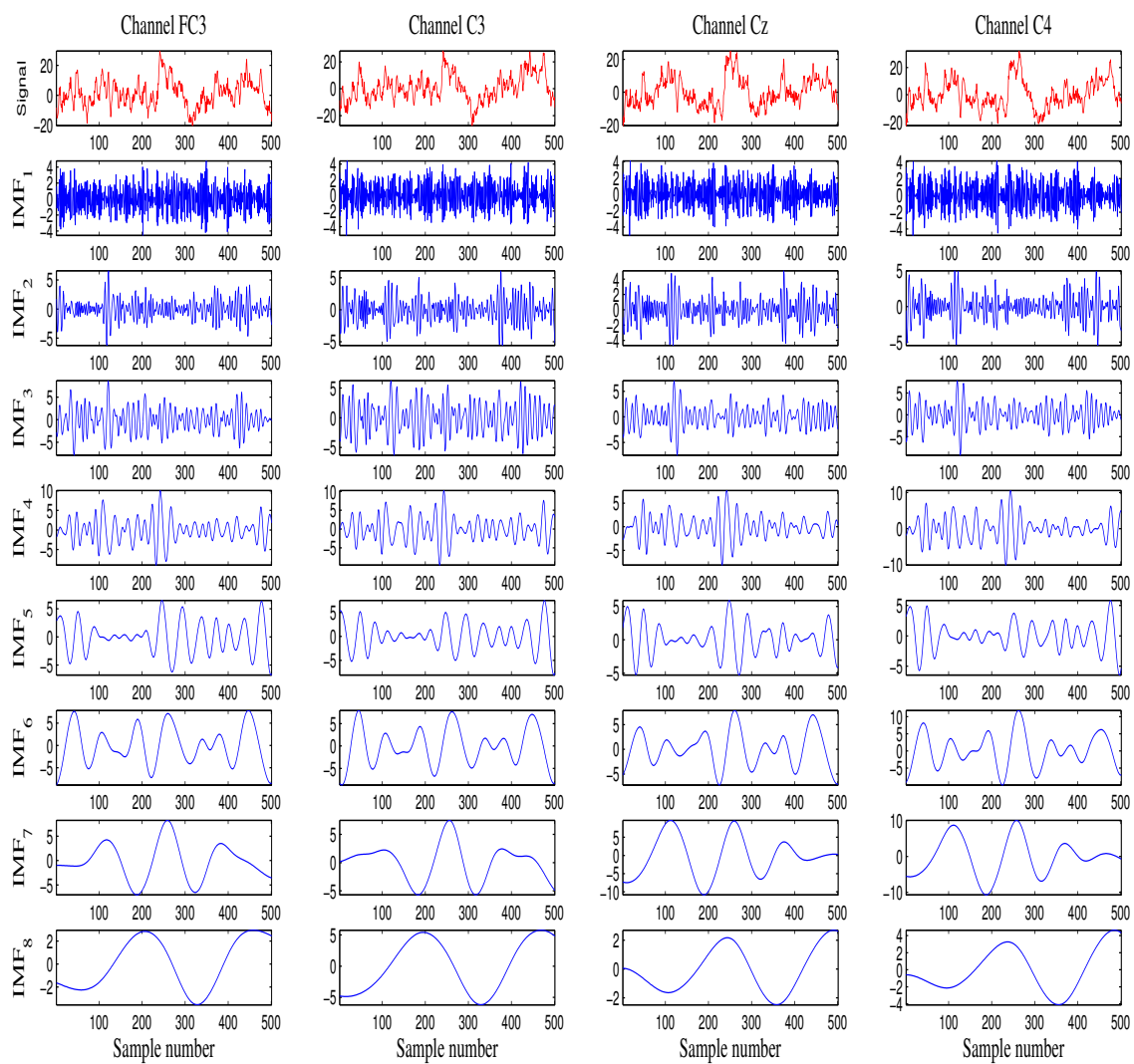

Fig. 4. The EEG signal corresponding to channels $\mathrm{FC} 3, \mathrm{C} 3, \mathrm{Cz}$, and $\mathrm{C} 4$ of the trial 1 of A08T for the RHMI task and its first nine IMFs.

considered from the subject A08's training session data A08T. Figure 3 demonstrates the LHMI tasks, raw EEG signals and its obtained IMFs whilst Figure 4 gives the RHMI EEG signal and its obtained IMFs. The statistical mean frequency measure has been computed for each of the IMFs obtained pertaining to LHMI and RHMI tasks. To get enhanced EEG signals pertaining to these MI tasks, the IMFs are first identified based on mean frequencies which lie in the frequency range $4-33$ $\mathrm{Hz}$ [12]. This frequency range comprises the mu $(\mu)$ band and beta $(\beta)$ band. These bands play a critical role in the identification of MI EEG signals [5], [36], [12].

Figure 5 and Figure 6 display the feature distribution of four features. The box plot in Fig. 5 represents the four features using the Kruskal-Wallis test with the MEMDBF method. To show the effect of the proposed method on the available features, feature separability is evaluated using the Wilcoxon test method for the LHMI and RHMI tasks. The features are arranged in decreasing order of class separability. The proposed preprocessing method has thus helped achieve statistically significant improvement in feature separability $(p<0.005)$ in training session for the LHMI and RHMI tasks. Figure 6 displays the same four features from the raw EEG signals giving $p$-values of $0.0250,0.3816,0.1572$, and 0.0046 . These $p$-values reveal the fact that the two features are not significantly different in their feature distribution for the LHMI and RHMI tasks. However, with the proposed pipeline, the $p$-values show a statistically significant difference in feature distribution for all four features. The subject A01 was used for computing results. The non-parametric Wilcoxon test is used for ranking the four features.

Table 1 illustrates the specifics of the rejected trials in the evaluation session from each subject marked with the event 1023 [35]. Subjects A04 and A06 have maximum number of the rejected trials. Subject A04 has a total 28 rejected trials while Subject A06 gives a total of 36 rejected trials. The rejected trials pertaining to right hand and left hand MI tasks across all nine subjects are as follow: right hand 58 trials and left hand 55 trials respectively. A subject specific rejected trials across all the nine subjects can be obtained from Table I.

TABLE I

REJECTED TRIALS FROM ALL SUBJECTS

\begin{tabular}{cccccc}
\hline Subject & $\begin{array}{c}\text { Total } \\
\text { Trials }\end{array}$ & $\begin{array}{c}\text { Correct } \\
\text { Trials }\end{array}$ & $\begin{array}{c}\text { Rejected } \\
\text { Trials }\end{array}$ & $\begin{array}{c}\text { Left } \\
\text { hand }\end{array}$ & $\begin{array}{c}\text { Right } \\
\text { hand }\end{array}$ \\
\hline A01 & 144 & 141 & 3 & 1 & 2 \\
A02 & 144 & 142 & 2 & 1 & 1 \\
A03 & 144 & 137 & 7 & 5 & 2 \\
A04 & 144 & 116 & 28 & 13 & 15 \\
A05 & 144 & 135 & 9 & 2 & 7 \\
A06 & 144 & 108 & 36 & 19 & 17 \\
A07 & 144 & 140 & 4 & 1 & 3 \\
A08 & 144 & 134 & 10 & 6 & 4 \\
A09 & 144 & 130 & 14 & 7 & 7 \\
\hline
\end{tabular}




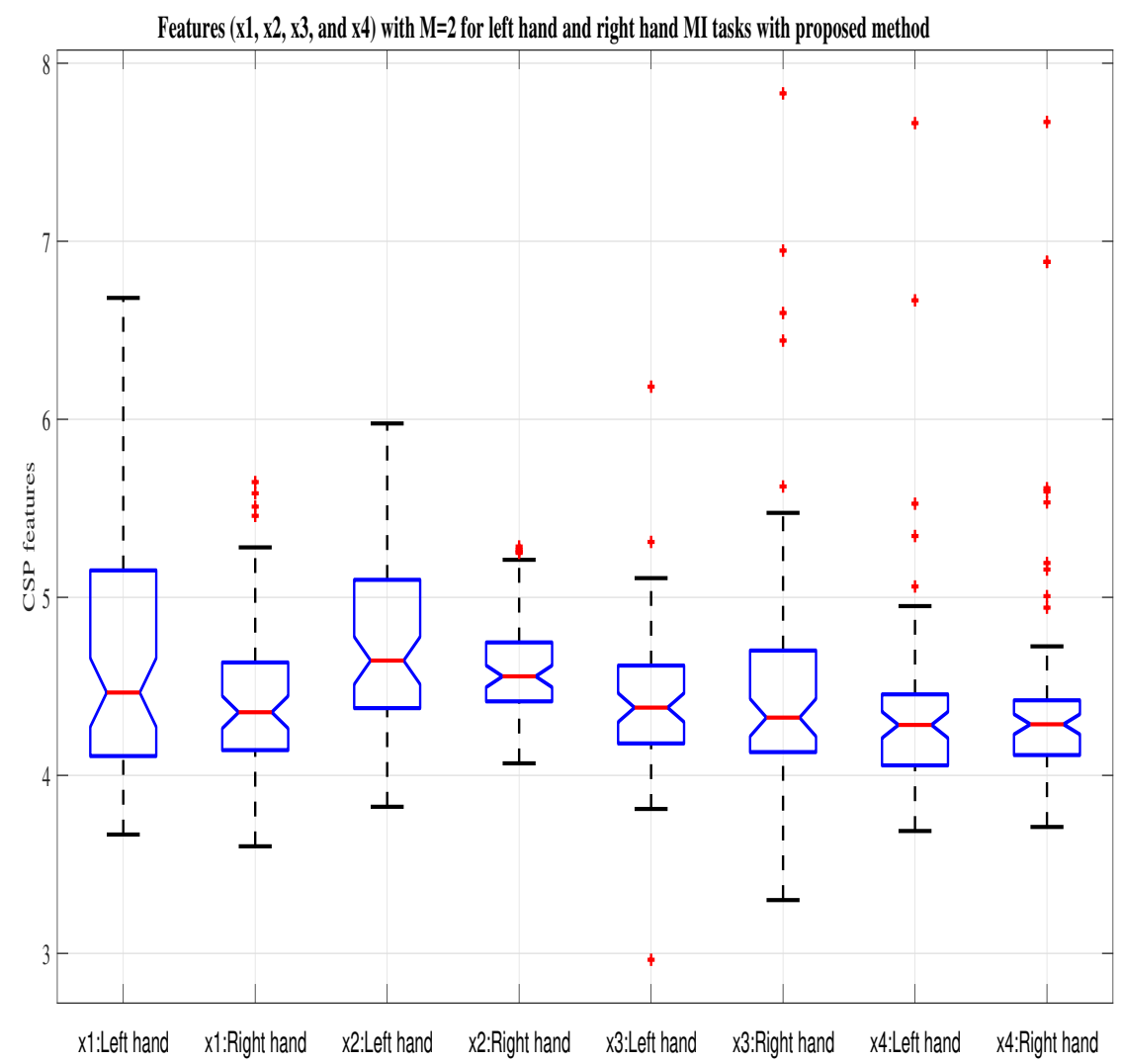

Fig. 5. The box plot displays the calculated four features using MEMDBF in the training session for left hand and right hand MI tasks.

Table III shows the classification accuracy and precision for the BCI competition IV dataset $2 \mathrm{~A}$ obtained using LDA classifier and SVM [37] classifier (linear kernel) with the proposed method. This method provides enhanced EEG signals for all A01-A09 subjects as compared to raw EEG signals, EMD based filtering and DWT based filtering, across two sessions namely, the training and evaluation sessions. This pre-processing step has helped to achieve a classification accuracy of $79.19 \%$ with LDA classifier and $79.18 \%$ with SVM classifier (linear kernel) in the evaluation session. Moreover, the classification accuracy was computed using other kernel functions as well such Additionally, the precision obtained is 79.88 and 80.22 using LDA and SVM classifier respectively.

Table III presents the comparison of the classification results obtained using the proposed method, raw EEG signals and other filtering techniques such as EMD based filtering and DWT based filtering. With the enhanced EEG signals using the MEMDBF method, the group average of classification accuracy improved by $6.68 \%$ as compared to raw EEG signals, $6.87 \%$ as compared to EMD based filtering and $11.49 \%$ as compared to DWT based filtering across all subjects considering both training and evaluation sessions. Evaluation accuracy has been computed with a classifier model created using $100 \%$ training session data. The results computed in the training session clearly depict that the average of the classification accuracy improved by $4.01 \%(p=0.0742)$ with a standard deviation of 11.86 with the MEMDBF-CSP method compared to the raw EEG signals considering the same features. The proposed method also helped to achieve an average group improvement of $>5 \%$ as compared to DWT based filtering in the training session. Notably, eight of the nine subjects have improved in classification accuracy in the evaluation session when compared to other filtering techniques and raw signals. Also the group average of classification accuracy across all nine subjects has improved by $>9 \%(p=0.0078)$ with raw EEG signals, $>13 \%(p=0.0039)$ with EMD based filtering and $>17 \%(p=0.0039)$ with DWT based filtering respectively. The proposed method was able to select the IMFs which were contributing to the specific bands.

With the proposed method, the difference between accuracies obtained in the training session and evaluation session have been very minimal $(>3 \%)$. As discussed, the training session accuracies have been computed using a five-fold crossvalidation mechanism. Evaluation accuracies in Table [III has been computed by creating a learning model with $100 \%$ of the training session data. Subjects A01, A03, A08, and A09 have obtained greater classification accuracy in the evaluation session as compared to the training session accuracies. In the column Evaluation, Subjects A02, A04, A05, A06, and A07 have a difference of $<8.5 \%$ in terms of classification accuracy across training and evaluation sessions. Thus, the results clearly show the proposed pipeline has helped to 


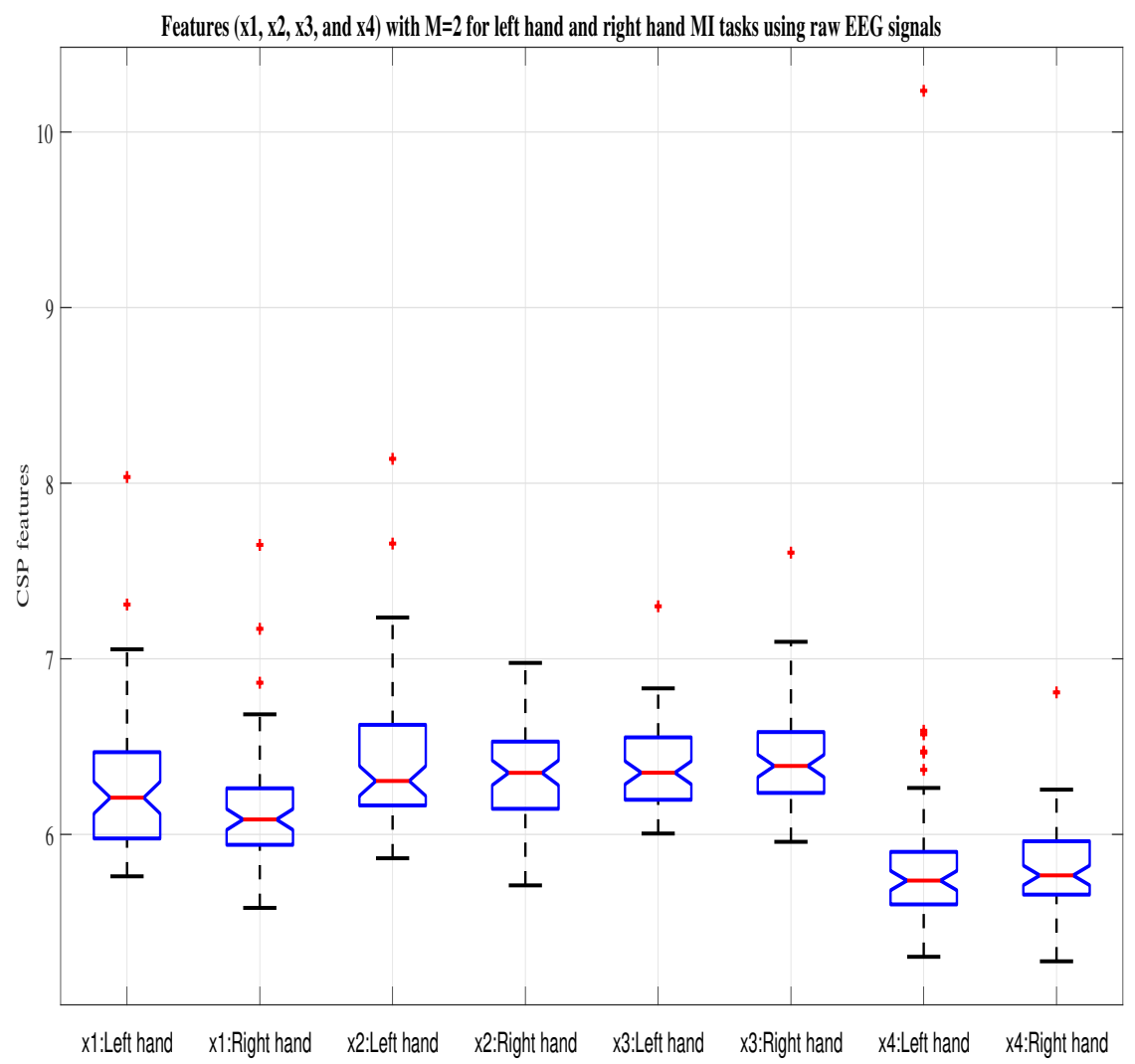

Fig. 6. The box plot reveals that the same four features from the raw EEG signals are not statistically significant in terms of separability with $p$-values $(0.0250,0.3816,0.1572$ and 0.0046$)$.

TABLE II

CLASSIFICATION ACCURACIES (IN \%) OBTAINED WITH THE PROPOSED MEMDBF METHOD AND RAW EEG SIGNALS BY LDA AND SVM CLASSIFIERS EVALUATED ON BCI COMPETITION IV DATASET 2A.

\begin{tabular}{|c|c|c|c|c|c|c|c|c|}
\hline \multirow{4}{*}{ Subject } & \multicolumn{8}{|c|}{ MEMDBF-CSP } \\
\hline & \multicolumn{4}{|c|}{ LDA } & \multicolumn{4}{|c|}{ SVM(Linear Kernel) } \\
\hline & \multicolumn{2}{|c|}{ Accuracy } & \multicolumn{2}{|c|}{ Precision } & \multicolumn{2}{|c|}{ Accuracy } & \multicolumn{2}{|c|}{ Precision } \\
\hline & Training & Evaluation & Training & Evaluation & Training & Evaluation & Training & Evaluation \\
\hline$\overline{\mathrm{A} 01}$ & 90.28 & 90.78 & 88.7 & 93.94 & 89.48 & 91.49 & 100 & 94.03 \\
\hline A02 & 65.28 & 57.75 & 65.16 & 55.45 & 61.9 & 59.86 & 76.92 & 57.95 \\
\hline $\mathrm{A} 03$ & 93.75 & 97.08 & 93.35 & 97.01 & 90.96 & 94.89 & 84.62 & 96.88 \\
\hline A04 & 74.31 & 70.69 & 69.81 & 68.66 & 72.11 & 70.69 & 57.14 & 75.51 \\
\hline A05 & 68.06 & 61.48 & 66.74 & 65 & 59.04 & 60.74 & 57.89 & 66.67 \\
\hline A06 & 78.47 & 70.37 & 78.64 & 69.09 & 73.62 & 67.59 & 76.92 & 66.07 \\
\hline A07 & 79.86 & 72.14 & 78.59 & 75.81 & 78.53 & 75 & 80 & 69.57 \\
\hline A08 & 97.22 & 97.76 & 95.14 & 95.65 & 96.57 & 97.76 & 100 & 97.01 \\
\hline A09 & 93.75 & 94.62 & 93.1 & 98.33 & 93.03 & 94.62 & 92.86 & 98.33 \\
\hline Average & 82.33 & 79.19 & 81.03 & 79.88 & 79.47 & 79.18 & 80.71 & 80.22 \\
\hline Std & 11.86 & 15.85 & 11.98 & 16.42 & 13.8 & 15.49 & 15.86 & 16.18 \\
\hline
\end{tabular}


TABLE III

CLASSIFICATION ACCURACIES (IN \%) OBTAINED WITH THE PROPOSED MEMDBF METHOD AND RAW EEG SIGNALS B Y LDA CLASSIFIER EVALUATED ON BCI COMPETITION IV DATASET $2 \mathrm{~A}$.

\begin{tabular}{ccccccccc}
\hline \multirow{2}{*}{ Subject } & \multicolumn{2}{c}{ MEMDBF-CSP } & \multicolumn{2}{c}{ Raw EEG Signal } & \multicolumn{2}{c}{ EMD-CSP } & \multicolumn{2}{c}{ DWT-CSP } \\
& Training & Evaluation & Training & Evaluation & Training & Evaluation & Training & Evaluation \\
\hline A01 & 90.28 & 90.78 & 72.27 & 69.44 & 84.01 & 68.79 & 70.17 & 53.19 \\
A02 & 65.28 & 57.75 & 63.21 & 50 & 73.62 & 49.3 & 70.81 & 50 \\
A03 & 93.75 & 97.08 & 91.65 & 90.28 & 89.48 & 81.75 & 76.96 & 64.96 \\
A04 & 74.31 & 70.69 & 71.58 & 59.03 & 77.14 & 54.31 & 70.81 & 53.45 \\
A05 & 68.06 & 61.48 & 67.92 & 50 & 74.39 & 51.85 & 73.67 & 51.85 \\
A06 & 78.47 & 70.37 & 67.99 & 54.86 & 77.73 & 58.33 & 71.57 & 49.07 \\
A07 & 79.86 & 72.14 & 86.18 & 65.28 & 75.02 & 45 & 82.66 & 60.71 \\
A08 & 97.22 & 97.76 & 95.19 & 97.92 & 95.12 & 92.54 & 86.89 & 80.6 \\
A09 & 93.75 & 94.62 & 88.86 & 91.67 & 93.12 & 88.46 & 90.91 & 88.46 \\
\hline Average & 82.33 & 79.19 & 78.32 & 69.83 & 82.18 & 65.59 & 77.16 & 61.37 \\
Std & 11.86 & 15.85 & 12.04 & 18.82 & 8.47 & 17.95 & 7.8 & 14.22 \\
$\boldsymbol{p}$-value & & & & 0.0078 & & 0.0039 & & 0.0039 \\
\hline
\end{tabular}

TABLE IV

COMPARISON OF CLASSIFICATION ACCURACIES (\%) OBTAINED WITH THE PROPOSED MEMDBF METHOD AND OTHER STATE-OF-THE-ART METHODS EVALUATED ON BCI COMPETITION IV DATASET 2 A.

\begin{tabular}{ccccc}
\hline Subject & MEMDBF-CSP & Method-1 & Method-2 & Method-3 \\
\hline A01 & $\mathbf{9 0 . 7 8}$ & 88.89 & 90.28 & 90.28 \\
A02 & $\mathbf{5 7 . 7 5}$ & 51.39 & 54.17 & 57.64 \\
A03 & $\mathbf{9 7 . 0 8}$ & 96.53 & 93.75 & 95.14 \\
A04 & $\mathbf{7 0 . 6 9}$ & 70.14 & 64.58 & 65.97 \\
A05 & $\mathbf{6 1 . 4 8}$ & 54.86 & 57.64 & 61.11 \\
A06 & 70.37 & $\mathbf{7 1 . 5 3}$ & 65.28 & 65.28 \\
A07 & 72.14 & $\mathbf{8 1 . 2 5}$ & 62.5 & 61.11 \\
A08 & $\mathbf{9 7 . 7 6}$ & 93.75 & 90.97 & 91.67 \\
A09 & $\mathbf{9 4 . 6 2}$ & 93.75 & 85.42 & 86.11 \\
\hline Average & 79.19 & 78.01 & 73.84 & 74.92 \\
Std & 15.85 & 17.01 & 15.93 & 15.42 \\
$\boldsymbol{p}$-value & & 0.2852 & 0.0039 & 0.0039 \\
\hline
\end{tabular}

counteract the inherent intersession non-stationarity present in the EEG signals. This difference in the classification accuracy across evaluation sessions in all subjects may be accounted with the adaptive techniques/ transfer learning mechanisms.

Table IV presents the comparison of classification accuracy values calculated with the MEMDBF-CSP method and other comparable works in the literature. The proposed MEMDBFCSP has shown comparable performance with one approach reported in [38] and substantial improvement when compared to other research works reported in [39]. The superior average classification accuracy has been achieved across nine subjects in comparison to results reported by four most recent advanced methods. The method-1 [38] reported 78.01\% ( $p=0.2852)$, method-2 [39] obtained $73.84 \%(p=0.0039)$ and method-3 [39] reported $74.92 \%(p=0.0039)$. The Wilcoxon signed rank test has been used to compute the $p$-values. These methods investigated the same two-class classification problem to classify the LHMI and RHMI tasks but there is a slight variation in the number of channels. Using the method-1, twenty-two channels raw EEG signal was bandpass-filtered between $8-30 \mathrm{~Hz}$ and further the CSP features with a number of components $(n c=3)$ were extracted. Thereafter, the features set was calculated by taking the $\log$ variance of three pairs of selected filters. Finally, they classified the feature set by an LDA classifier [38]. The method-1 considered all twenty-two channels $(n c h=22)$ to compute the classification accuracy while comparable results are obtained with the MEMDBF-CSP method using only fifteen channels $(n c h=15)$. Method-2 and method-3 used only ten channels $(n c h=10)$ for the study. They extracted CSP features from the bandpass filtered ten EEG channels and further only 1 component $(n c=1)$ was selected from the CSP features. They detected the covariate shift in the feature matrix and then applied adaptive learning and transductive learning to adapt to the covariate shifts [39]. Their method performs adaptation by updating the classifier in the evaluation stage. More details can be obtained from [39]. The MEMDBF-CSP thus demonstrates a tangible improvement in classification accuracy for seven of the nine subjects as marked in boldface in Table IV

\section{CONCLUSION}

A pipeline, namely, MEMDBF-CSP has been proposed to enhance performance in MI-based BCI with a minimal number of channels. This pipeline has the MEMDBF method as a preprocessing step which is an extension of the MEMD. In the second step of this pipeline, CSP features have been implemented for enhancing the performance of a two-class MI-based BCI. The key idea in the proposed pipeline is that at the preprocessing stage, MEMD based filtering removes inherent non-stationarity present in EEG signals to some extent whilst filtering artifacts and noise. The enhanced EEG signals have zero mean. There is no complexity introduced at the feature extraction step or the classification step. A highly significant performance has been obtained in MI-based BCI simply by enhancing the EEG signals at the pre-processing stage. A selection of multiple IMFs based on the median 
frequency measure which lie in the frequency range of the mu and beta bands, helped gain improvement in classification accuracy, while classifying LHMI and RHMI-based EEG signals as compared to raw EEG signals with CSP features. The classification accuracy obtained from this pipeline has shown significant improvement through both the training and the evaluation sessions across multiple subjects. The MEMDBF with CSP features has thus shown superior performance in classification accuracy not only when compared to raw EEG signals, but also when compared with similar advanced techniques such as adaptive learning. An improved feature separability was achieved using this pipeline. As a result, nonstationarity present in the EEG signal has been handled to a good extent. Future work may include proposing automated computational methods such as a genetic algorithm (GA) or particle swarm optimization (PSO) for selecting a subject specific channel combination or parameters, which may further increase performance. It may also be interesting to evaluate the performance of pre-processing stage with hidden Markov model (HMM) or long short-term memory (LSTM) networks.

\section{REFERENCES}

[1] J. R. Wolpaw, N. Birbaumer, D. J. McFarland, G. Pfurtscheller, and T. M. Vaughan, "Brain-computer interfaces for communication and control," Clinical Neurophysiology, vol. 113, no. 6, pp. 767-791, 2002.

[2] G. Pfurtscheller, C. Neuper, D. Flotzinger, and M. Pregenzer, "EEGbased discrimination between imagination of right and left hand movement," Electroencephalography and Clinical Neurophysiology, vol. 103, no. 6, pp. 642-651, 1997.

[3] G. Pfurtscheller, C. Brunner, A. Schlögl, and F. L. Da Silva, "Mu rhythm (de) synchronization and EEG single-trial classification of different motor imagery tasks," Neuroimage, vol. 31, no. 1, pp. 153-159, 2006.

[4] P. Herman, G. Prasad, T. M. McGinnity, and D. Coyle, "Comparative analysis of spectral approaches to feature extraction for EEG-based motor imagery classification," IEEE Transactions on Neural Systems and Rehabilitation Engineering, vol. 16, no. 4, pp. 317-326, 2008.

[5] V. Gandhi, G. Prasad, D. Coyle, L. Behera, and T. M. McGinnity, "Quantum neural network-based EEG filtering for a brain-computer interface," IEEE Transactions on Neural Networks and Learning Systems, vol. 25, no. 2, pp. 278-288, 2014.

[6] L. F. Nicolas-Alonso and J. Gomez-Gil, "Brain computer interfaces, a review," Sensors, vol. 12, no. 2, pp. 1211-1279, 2012.

[7] S. Lemm, B. Blankertz, G. Curio, and K.-R. Müller, "Spatio-spectral filters for improving the classification of single trial EEG," IEEE Transactions on Biomedical Engineering, vol. 52, no. 9, pp. 1541-1548, 2005.

[8] H. Zhang, H. Yang, and C. Guan, "Bayesian learning for spatial filtering in an EEG-based brain-computer interface," IEEE Transactions on Neural Networks and Learning Systems, vol. 24, no. 7, pp. 1049-1060, 2013.

[9] D. Coyle, "Neural network based auto association and time-series prediction for biosignal processing in brain-computer interfaces," IEEE Computational Intelligence Magazine, vol. 4, no. 4, pp. 47-59, 2009.

[10] D. Coyle, G. Prasad, and T. M. McGinnity, "A time-series prediction approach for feature extraction in a brain-computer interface," IEEE Transactions on Neural Systems and Rehabilitation Engineering, vol. 13, no. 4, pp. 461-467, 2005.

[11] _ _ "Faster self-organizing fuzzy neural network training and a hyperparameter analysis for a brain-computer interface," IEEE Transactions on Systems, Man, and Cybernetics, Part B: Cybernetics, vol. 39, no. 6, pp. 1458-1471, 2009

[12] P. Gaur, R. B. Pachori, H. Wang, and G. Prasad, "An empirical mode decomposition based filtering method for classification of motor-imagery EEG signals for enhancing brain-computer interface," in International Joint Conference on Neural Networks, 2015, pp. 1-7.

[13] R. Sharma, R. B. Pachori, and U. R. Acharya, "Application of entropy measures on intrinsic mode functions for the automated identification of focal electroencephalogram signals," Entropy, vol. 17, no. 2, pp. 669691,2015
[14] R. Sharma and R. B. Pachori, "Classification of epileptic seizures in EEG signals based on phase space representation of intrinsic mode functions," Expert Systems with Applications, vol. 42, no. 3, pp. 1106-1117, 2015.

[15] C. Park, D. Looney, A. Ahrabian, D. P. Mandic et al., "Classification of motor imagery BCI using multivariate empirical mode decomposition," IEEE Transactions on Neural Systems and Rehabilitation Engineering, vol. 21, no. 1, pp. 10-22, 2013.

[16] S. R. Davies and C. J. James, "Novel use of empirical mode decomposition in single-trial classification of motor imagery for use in braincomputer interfaces," in 35th Annual International Conference of the IEEE Engineering in Medicine and Biology Society, 2013, pp. 56105613.

[17] $ـ$, "Using empirical mode decomposition with spatio-temporal dynamics to classify single-trial motor imagery in BCI," in Engineering in Medicine and Biology Society (EMBC), 2014 36th Annual International Conference of the IEEE. IEEE, 2014, pp. 4631-4634.

[18] C. Park, M. Plank, J. Snider, S. Kim, H. C. Huang, S. Gepshtein, T. P. Coleman, and H. Poizner, "EEG Gamma Band Oscillations Differentiate the Planning of Spatially Directed Movements of the Arm Versus Eye: Multivariate Empirical Mode Decomposition Analysis," IEEE Transactions on Neural Systems and Rehabilitation Engineering, vol. 22, no. 5, pp. 1083-1096, 2014.

[19] S. Mallat, A wavelet tour of signal processing: the sparse way. Academic press, 2008.

[20] J. Kevric and A. Subasi, "Comparison of signal decomposition methods in classification of eeg signals for motor-imagery bci system," Biomedical Signal Processing and Control, vol. 31, pp. 398-406, 2017.

[21] D. Labate, F. Foresta, G. Occhiuto, F. C. Morabito, A. Lay-Ekuakille, and P. Vergallo, "Empirical mode decomposition vs. wavelet decomposition for the extraction of respiratory signal from single-channel ecg: A comparison," IEEE Sensors Journal, vol. 13, no. 7, pp. 2666-2674, 2013.

[22] P. Gaur, R. B. Pachori, H. Wang, and G. Prasad, "A multi-class EEGbased BCI classification using multivariate empirical mode decomposition based filtering and Riemannian geometry," Expert Systems with Applications, vol. 95, pp. 201-211, 2018.

[23] N. E. Huang, Z. Shen, S. R. Long, M. C. Wu, H. H. Shih, Q. Zheng, N.C. Yen, C. C. Tung, and H. H. Liu, "The empirical mode decomposition and the Hilbert spectrum for nonlinear and non-stationary time series analysis," in Proceedings of the Royal Society of London A: Mathematical, Physical and Engineering Sciences, vol. 454, no. 1971. The Royal Society, 1998, pp. 903-995.

[24] G. Pfurtscheller, C. Neuper, D. Flotzinger, and M. Pregenzer, "EEGbased discrimination between imagination of right and left hand movement," Electroencephalography and Clinical Neurophysiology, vol. 103, no. 6, pp. 642-651, 1997.

[25] Z. Wu and N. E. Huang, "Ensemble empirical mode decomposition: a noise-assisted data analysis method," Advances in adaptive data analysis, vol. 1, no. 01, pp. 1-41, 2009.

[26] N. E. Huang, M.-L. C. Wu, S. R. Long, S. S. Shen, W. Qu, P. Gloersen, and K. L. Fan, "A confidence limit for the empirical mode decomposition and Hilbert spectral analysis," in Proceedings of the Royal Society of London a: Mathematical, Physical and Engineering Sciences, vol. 459, no. 2037. The Royal Society, 2003, pp. 2317-2345.

[27] G. Rilling, P. Flandrin, P. Goncalves et al., "On empirical mode decomposition and its algorithms," in IEEE-EURASIP Workshop on Nonlinear Signal and Image Processing, vol. 3. IEEER, Grado, Italy, 2003, pp. 8-11.

[28] N. Rehman and D. P. Mandic, "Multivariate empirical mode decomposition," in Proceedings of The Royal Society of London A: Mathematical, Physical and Engineering Sciences. The Royal Society, 2009, p. rspa20090502.

[29] N. ur Rehman, C. Park, N. E. Huang, and D. P. Mandic, "EMD via MEMD: multivariate noise-aided computation of standard EMD," Advances in Adaptive Data Analysis, vol. 5, no. 02, p. 1350007, 2013.

[30] A. Phinyomark, S. Thongpanja, H. Hu, P. Phukpattaranont, and C. Limsakul, "The usefulness of mean and median frequencies in electromyography analysis," in Computational Intelligence in Electromyography Analysis-A Perspective on Current Applications and Future Challenges. InTech, 2012

[31] B. Blankertz, R. Tomioka, S. Lemm, M. Kawanabe, and K.-R. Muller, "Optimizing spatial filters for robust EEG single-trial analysis," Signal Processing Magazine, IEEE, vol. 25, no. 1, pp. 41-56, 2008.

[32] P. Gaur, R. B. Pachori, H. Wang, and G. Prasad, "A multivariate empirical mode decomposition based filtering for subject independent BCI," in 27th Irish Signals and Systems Conference (ISSC). IEEE, 2016, pp. 1-7. 
[33] K. K. Ang, Z. Y. Chin, C. Wang, C. Guan, and H. Zhang, "Filter bank common spatial pattern algorithm on BCI competition IV datasets $2 \mathrm{a}$ and 2b," Frontiers in Neuroscience, vol. 6, 2012.

[34] F. Lotte, M. Congedo, A. Lécuyer, and F. Lamarche, "A review of classification algorithms for EEG-based brain-computer interfaces," Journal of Neural Engineering, vol. 4, 2007.

[35] C. Brunner, R. Leeb, G. Müller-Putz, A. Schlögl, and G. Pfurtscheller, "BCI Competition 2008-Graz data set A," Institute for Knowledge Discovery (Laboratory of Brain-Computer Interfaces), Graz University of Technology, pp. 136-142, 2008.

[36] V. Gandhi, G. Prasad, D. Coyle, L. Behera, and T. M. McGinnity, "Evaluating quantum neural network filtered motor imagery brain-computer interface using multiple classification techniques," Neurocomputing, vol. 170, pp. 161-167, 2015.

[37] C. Cortes and V. Vapnik, "Support-vector networks," Machine learning, vol. 20, no. 3, pp. 273-297, 1995.

[38] F. Lotte and C. Guan, "Regularizing common spatial patterns to improve BCI designs: unified theory and new algorithms," IEEE Transactions on biomedical Engineering, vol. 58, no. 2, pp. 355-362, 2011.

[39] H. Raza, H. Cecotti, Y. Li, and G. Prasad, "Adaptive learning with covariate shift-detection for motor imagery-based brain-computer interface," Soft Computing, vol. 20, no. 8, pp. 3085-3096, 2016.

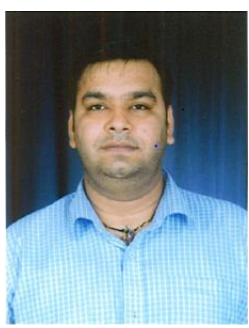

Pramod Gaur received the BE degree with honors in Computer Science and Engineering from University of Rajasthan, Jaipur, India in 2004, PGDIT from Indian Institute of Technology, Kharagpur, India in 2006, ME degree in Software Engineering from Birla Institute of Technology, Ranchi, India in 2008 and $\mathrm{PhD}$ in 2018 from Ulster University, UK. He is currently working as a research assistant in neuroimaging technology in Intelligent Systems Research Centre, Ulster University, UK. His research interest includes brain-computer Interface, analysis of nonstationary signals, machine learning.

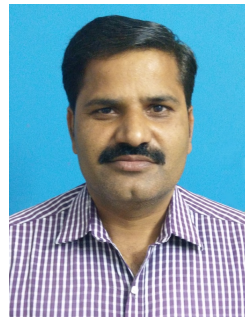

Ram Bilas Pachori received the B.E. degree with honours in Electronics and Communication Engineering from Rajiv Gandhi Technological University, Bhopal, India in 2001, the M.Tech. and Ph.D. degrees in Electrical Engineering from Indian Institute of Technology (IIT) Kanpur, Kanpur, India in 2003 and 2008, respectively. He worked as a Postdoctoral Fellow at Charles Delaunay Institute, University of Technology of Troyes, Troyes, France during 20072008. He served as an Assistant Professor at Communication Research Center, International Institute of Information Technology, Hyderabad, India during 2008-2009. He served as an Assistant Professor at Discipline of Electrical Engineering, IIT Indore, Indore, India during 2009-2013. He worked as an Associate Professor at Discipline of Electrical Engineering, IIT Indore, Indore, India during 20132017 where presently he has been working as a Professor since 2017. He is also an Associated Faculty with Discipline of Biosciences\& Biomedical Engineering at IIT Indore. He has been working as a Visiting Professor at School of Medicine, Faculty of Health and Medical Sciences, TaylorEs University, Subang Jaya, Malaysia since December 2018. He worked as a Visiting Scholar at Intelligent Systems Research Center, Ulster University, Northern Ireland, UK during December 2014. He is an Associate Editor of Biomedical Signal Processing and Control journal and an Editor of IETE Technical Review journal. He is a senior member of IEEE and a Fellow of IETE. He has more than 150 publications which include journal papers, conference papers, books, and book chapters. His publications have around 3500 citations, h index of 32, and i10 index of 70 (Google Scholar, January 2019). He has served on review boards for more than 85 scientific journals and served for scientific committees of various national and international conferences. His research interests are in the areas of biomedical signal processing, non-stationary signal processing, speech signal processing, signal processing for communications, computer-aided medical diagnosis, and signal processing for mechanical systems.

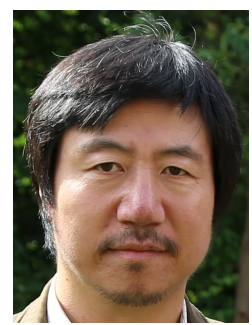

Hui Wang received the BSc degree in computer science and MSc degree in artificial intelligence both from Jilin University, China, and the PhD degree in artificial intelligence from the University of Ulster. $\mathrm{He}$ is a Professor at the School of Computing and Mathematics, University of Ulster, United Kingdom. His research interests include pattern recognition, machine learning, data/text mining, financial data mining, uncertainty reasoning, spatial reasoning, and information retrieval.

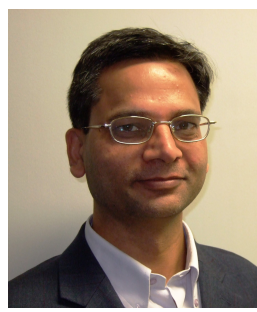

Girijesh Prasad (M'98-SM'07) received a BTech degree in Electrical Engineering from REC (now NIT) Calicut, India in 1987, an MTech degree in Computer Science \& Technology from University of Roorkee (now IIT) Roorkee, India in 1992, and a $\mathrm{PhD}$ degree from Queens University, Belfast, UK in 1997. Currently he is Professor of Intelligent Systems in the School of Computing \& Intelligent Systems, Ulster University, Derry Londonderry, UK. As an executive member of Intelligent Systems Research Centre at Ulster, he leads the Neural Systems and Neuro-technology team. He is the Director of Northern Ireland Functional Brain Mapping facility for MEG studies. His research interests are in computational intelligence, brain modelling, brain-computer interfaces and neuro-rehabilitation, and assistive technology. He has published over 210 research papers in international journals, books, and conference proceedings.

Prof. Prasad is a Chartered Engineer and a fellow of the IET. He is a founder member of IEEE SMC TCs on Brain-Machine Interface Systems and Evolving Intelligent Systems. 